\title{
Origin of X-ray Spectral Variation and the Seemingly Broad Iron-Line Spectral Feature in Seyfert Galaxies
}

\author{
Ken Ebisawa ${ }^{1,2}$, Naoki Iso ${ }^{1,2}$, Takehiro Miyakawa ${ }^{3}$, Hajime Inoue ${ }^{1}$ \\ ${ }^{1}$ Institute of Space and Astronautical Science (ISAS), Japan Aerospace Exploration Agency (JAXA), 3-1-1 Yoshinodai, \\ Chuo-ku, Sagamihara, Kanagawa 252-5210 \\ ${ }^{2}$ Department of Astronomy, Graduate School of Science, The University of Tokyo, 7-3-1 Hongo, Bunkyo-ku, Tokyo \\ 113-0033 \\ ${ }^{3}$ Tsukuba Space Center (TKSC), Japan Aerospace Exploration Agency (JAXA), 2-1-1 Sengen, Tsukuba-shi, Ibaraki 305- \\ 8505, Japan
}

Corresponding author: ebisawa@isas.jaxa.jp

\begin{abstract}
We present systematic X-ray data analysis of the Seyfert galaxies observed by Suzaku to study origin of their hard X-ray $(2-40 \mathrm{keV})$ variations. In particular, we examine if the "Variable Partial Covering (VPC) model" proposed by Miyakawa, Ebisawa and Inoue (2012), which was successful to explain spectral variations of MCG-6-30-15, is also valid for other Seyfert galaxies or not. In this model, intrinsic X-ray luminosity of the AGN is not significantly variable, and most observed flux and spectral variations are caused by change of the geometrical covering fraction of the extended X-ray source by ionized absorbing clouds in the line of sight. We found that the observed flux and spectral variations of 20 targets in addition to MCG-6-30-15 are successfully explained by the VPC model. The transmitted spectral component through the absorbing clouds has a characteristics spectral feature of the ionized iron K-edge, which is considered to be the origin of the seemingly broad iron-line feature commonly observed in Seyfert galaxies. Variation of the partial covering fraction of the constant X-ray luminosity source causes such an anti-correlation between the direct (non-obscured) component and the transmitted (obscured) component, that cancels their variations each other. The cancellation works most effectively at the energy band where intensities of the two components are the closest to each other, namely, just below the iron K-edge. This explains the significantly small fractional variations in the iron K-energy band, another well-known observational characteristic of Seyfert galaxies.
\end{abstract}

Keywords: black holes - Active Galactic Nuclei - accretion disk - warm absorbers - partial covering - X-rays - individual: MCG-6-30-15.

\section{Introduction}

Recently, Miyakawa, Ebisawa and Inoue (2012) (MEI2012 hereafter) proposed a "variable partial covering (VPC) model " to explain X-ray spectral variation of Seyfert galaxies. In this model, the original Xray luminosity of the AGN is not significantly variable, and the apparent $\mathrm{X}$-ray variation is primarily caused by variation of the geometrical covering fraction of the extended X-ray source by the intervening clouds having internal ionization structures. MEI2012 applied the VPC model to Suzaku observations of MCG-6-30-15, and successfully explained not only the small fractional variation in the iron energy band, but also the entire intensity and spectral variations in $1-40 \mathrm{keV}$ (see also Inoue, Miyakawa and Ebisawa 2011).

It is interesting to examine if the VPC model is also valid for other Seyfert galaxies, and intrinsic X-ray lu- minosities of the Seyfert galaxies in general are significantly variable or not. In the present paper, we explore the Suzaku archive to select Seyfert galaxies which show similar X-ray spectral characteristics to MCG-6-30-15, and apply the VPC model to see if their X-ray intensity and spectral variations are explained by this model or not.

\subsection{Data selection}

We chose data from the Suzaku public archive, as of 2011 October 5. Our main purpose is to study X-ray intensity and spectral variations of the Seyfert galaxies, which have similar spectral characteristics to MCG-6$30-15$, in particular in the iron K-energy band. Therefore, we selected only targets which are classified as Seyfert galaxies, and known to show the seemingly broad iron K-line feature or a hint of that. 
Origin of X-ray Spectral Variation and the Seemingly Broad Iron-Line Spectral Feature in Seyfert Galaxies

To study spectral variations efficiently, we chose only the observations which satisfy the following conditions; (1) long enough total exposure time (> $60 \mathrm{ksec})$ for an observation, (2) bright enough that the total accumulate counts in an observation are more than 50,000 counts in $0.2-10 \mathrm{keV}$, and (3) the sources are variable more than $10 \%$ in $4-10 \mathrm{keV}$ in an obseravtion. Thus, we selected 27 observations for 25 targets (using 50 sequences in total). Figure 1 shows two examples of the $0.2-12.0 \mathrm{keV}$ light curves (NGC3516 and NGC4051) among the 27 observations.

\section{Data Analysis and Results}

\subsection{Time-averaged spectra}

Firstly, we analyze the time-averaged spectra of the 27 observations from the 25 sources. We apply the " 3 component model" proposed by MEI2012. The model is represented as

$$
F=W_{H} W_{L}\left(N_{1}+W_{2} N_{2}\right) P+R P N_{3}+I_{\mathrm{Fe}},
$$

where $P$ is the intrinsic cut-off power-law spectrum, $N_{1}$ and $N_{2}$ are the direct power-law normalization and the absorbed power-law normalization, respectively. $W_{H}$, $W_{L}$, and $W_{2}$ represents the transmissions due to highionized warm absorber, low-ionized warm absorber, and partial heavy absorber $\left(N_{\mathrm{H}}>10^{24} \mathrm{~cm}^{-2}\right)$, respectively. Each warm-absorber has two parameters, the hydrogen column-density, $N_{H}$, and the ionization parameter, $\xi$, such that $W_{H}=\exp \left(-\sigma\left(\xi_{H}\right) N_{H, H}\right), W_{L}=$ $\exp \left(-\sigma\left(\xi_{L}\right) N_{H, L}\right)$ and $W_{2}=\exp \left(-\sigma\left(\xi_{2}\right) N_{H, 2}\right)$, where $\sigma(\xi)$ means the photo-absorption cross-section. $R$ and $N_{3}$ are the reflection albedo and reflection normalization by the neutral accretion disk, $I_{\mathrm{Fe}}$ is a narrow iron $\mathrm{K} \alpha$ emission line, respectively. The interstellar extinction is also included in the model fitting, but not explicitly shown in equation (1). For details of the spectral model, see MEI2012.

Consequently, we found 22 time-averaged spectra out of 20 sources are successfully represented with the 3 -component model.

\subsection{Intensity-sliced spectra}

Next, we study spectral variations within the 22 observations (out of 20 sources), average spectra of which were successfully fitted by the 3-component model in the previous section. We examine if the "variable partial covering (VPC) model" proposed by MEI2012 may explain the observed spectral variations or not. To prepare, we briefly review the VPC model in the following: Let's define the "total normalization", $N$, and the partial covering fraction, $\alpha$, so that

$$
N=N_{1}+N_{2}
$$

and

$$
N_{1}=(1-\alpha) N, N_{2}=\alpha N .
$$

Detailed study of spectral variations of MCG-6-30-15 by MEI2012 suggests that the low-ionized warm absorber, which is optically thin, is associated with the same heavy absorber causing the partial covering (see Figure 12 in MEI2012), so that

$$
\begin{aligned}
W_{L} & \approx 1-\sigma\left(\xi_{L}\right) N_{H, L} \approx 1-\alpha \sigma\left(\xi_{L}\right) N_{H, L}^{(f i x e d)} \\
& \approx(1-\alpha)+\alpha \exp \left(-\sigma\left(\xi_{L}\right) N_{H, L}^{(f i x e d)}\right),
\end{aligned}
$$

where $N_{H, L}^{(\text {fixed })}$ is the fixed amount of the column density of the low-ionized absorber associated with the absorbing cloud, presumably as an outer-envelope. Consequently, the 3 -component model (1) is rewritten as "double partial covering", such that

$$
\begin{gathered}
F=\exp \left(-\sigma\left(\xi_{H}\right) N_{H, H}\right) \times \\
\left(1-\alpha+\alpha \exp \left(-\sigma\left(\xi_{L}\right) N_{H, L}^{f i x e d}\right)\right) \times \\
\left(1-\alpha+\alpha \exp \left(-\sigma\left(\xi_{2}\right) N_{H, 2}\right)\right) P N \\
+R P N_{3}+I_{\mathrm{Fe}} .
\end{gathered}
$$

Following MEI2012, we create intensity-sliced spectra, and examine the spectral model (5). For each observation, we define four intensity ranges in the $0.2-$ $12.0 \mathrm{keV}$ light curve so that the photon counts for each intensity range be approximately equal, and create the four intensity-sliced energy spectra. The red dotted horizontal lines in Figure 1 indicate the intensity boundaries for each observation.

We try to fit the four intensity-spectra in $2-40$ $\mathrm{keV}$ simultaneously only making $\alpha$ variable. As a result, we successfully fitted the intensity-sliced spectra of the 20 observations of 18 sources only varying $\alpha$. Two examples are shown in Figure 3. The rest two sources requires $N$ to be slightly variable.

\subsection{Time-sliced spectra}

Next, we see that time sequences of the energy spectra are also explained by the VPC model with timevarying partial covering fractions. In the XIS lightcurves, we define boundaries of the time-sliced spectra every $2.3 \times 10^{4} \mathrm{sec}$, which corresponds to four Suzaku orbital periods. The blue vertical dotted lines in Figure 1 show the time-boundaries for each observation, and we made energy spectra from each time bin. Numbers of the time-sliced spectra in a single observation are from 6 (4C 74.26) to 28 (NGC 4051). For each observation, a time-series of the spectra are fitted simultaneously with the VPC model in Eq. (5). We first try to fit the spectra only allowing the partial covering fraction $\alpha$ to vary. If not successful (i.e., $\chi_{r}^{2}>1.2$ ), the total normalization $N$ is varied in addition. 

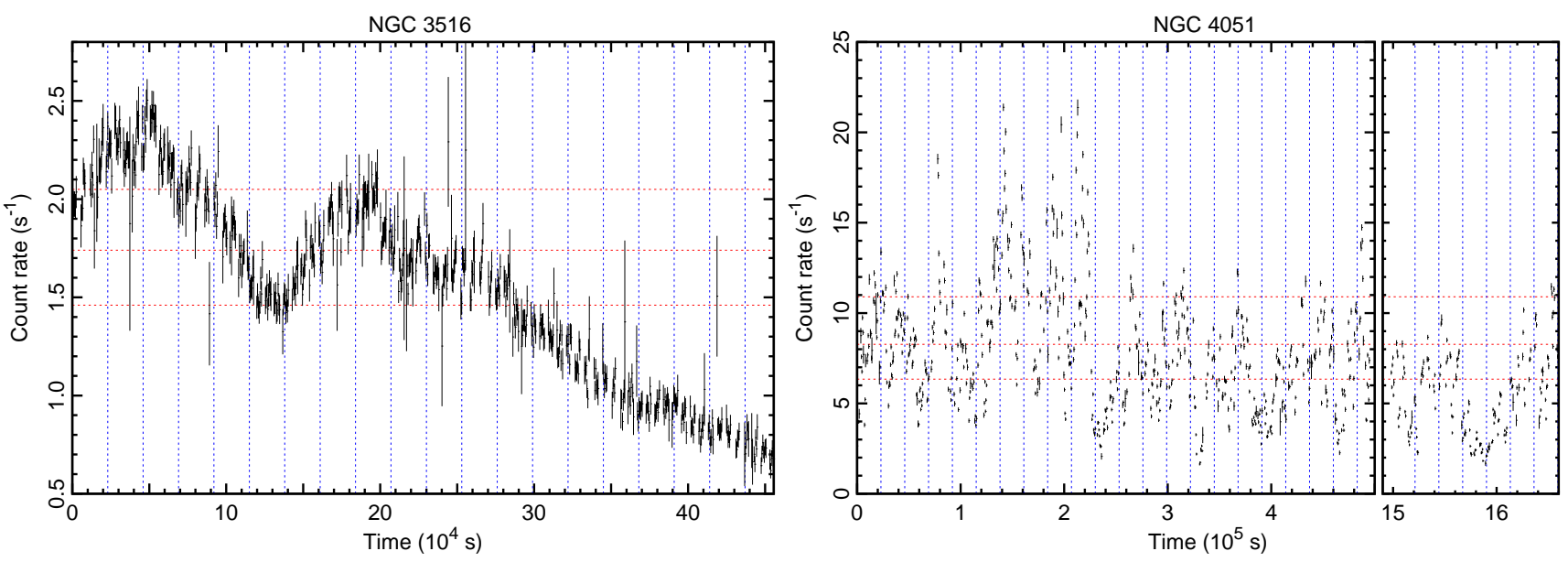

Figure 1: XIS light curves of NGC3516 and NGC4051 in $0.2-12.0 \mathrm{keV}$. The count rate is binned with $512 \mathrm{~s}$. Horizontal red-dotted lines show the count rate intervals with which the intensity-sliced spectra were made (section 2.2). Vertical blue-dotted lines show the time intervals with which the time-sliced spectra were made (section $2.3)$.
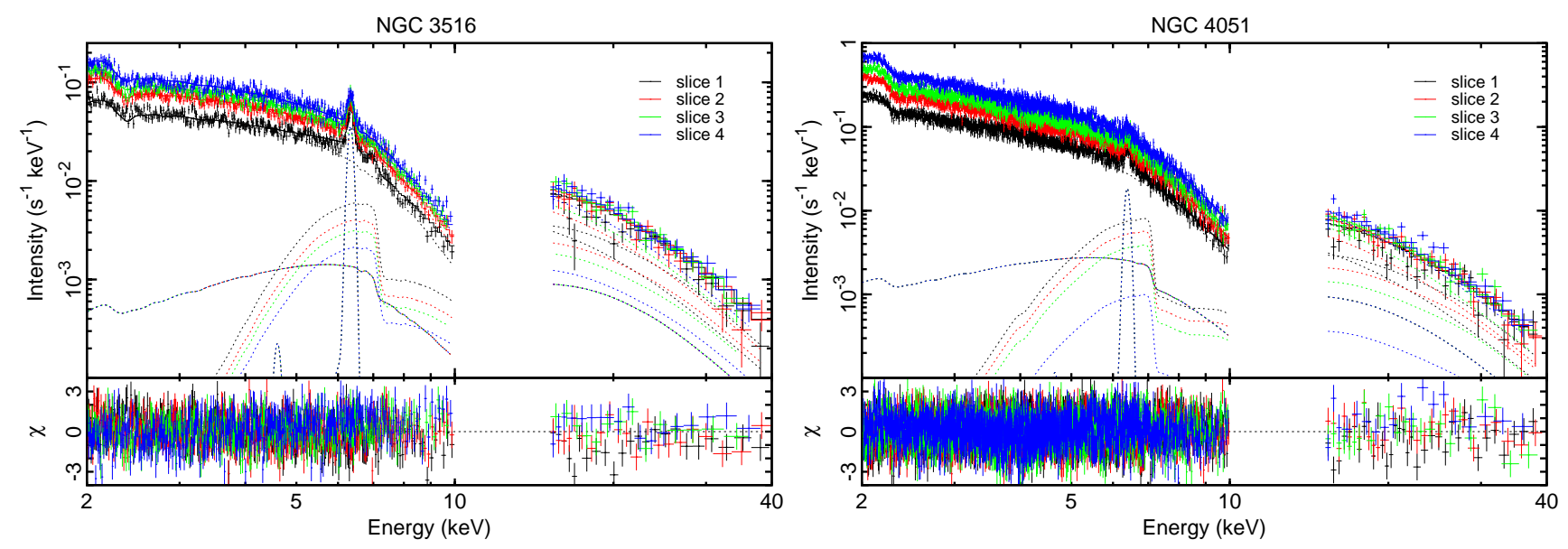

Figure 2: Model fitting results of the intensity-sliced XIS and PIN spectra for NGC3516 and NGC4051. For each observation, the four intensity-sliced spectra are made with approximately equal counts, and the four spectra are fitted simultaneously only varying the partial covering fraction, $\alpha$. The data and the best-fit power-law model are in the upper panel, while the residuals are in the lower panel.

As a result, 21 observations from 19 sources were explained only varying $\alpha$. Figure 3 shows variation of the observed counting rates in $0.2-12 \mathrm{keV}$ and the partial covering fraction for two examples among the $21 \mathrm{ob}-$ servations. Anti-correlation between the counting rate and the partial covering fraction is obvious, that means the apparent flux increase/decrease of these sources are caused by decrease/increase of the partial covering fraction of the constant luminosity X-ray sources.

We notice variability in the optical and UV continuum and lines is often seen to be correlated with observed X-ray variability, while our model requires that the X-ray intrinsic luminosity hardly changes. This suggests that the osculation of the intrinsic X-ray source by the intervening absorbing clouds takes place just inbetween the central black hole and the optical/UV emitting regions, so that the optical/UV lights reflect the variable absorbed X-ray fluxes.

\subsection{Root Mean Square(RMS) spectra}

Finally, we calculate Root Mean Square (RMS) spectra for the 22 observations using the XIS data in order to investigate time variation in the iron energy band. We use the same time-series of the spectra used in the previous section as well as the best-fit models. Namely, RMS spectra are calculated with a bin-width of $2.3 \times 10^{4} \mathrm{sec}$.

We divided the time-sliced spectra into 15 energy band, and computed the RMS spectra. We show two 

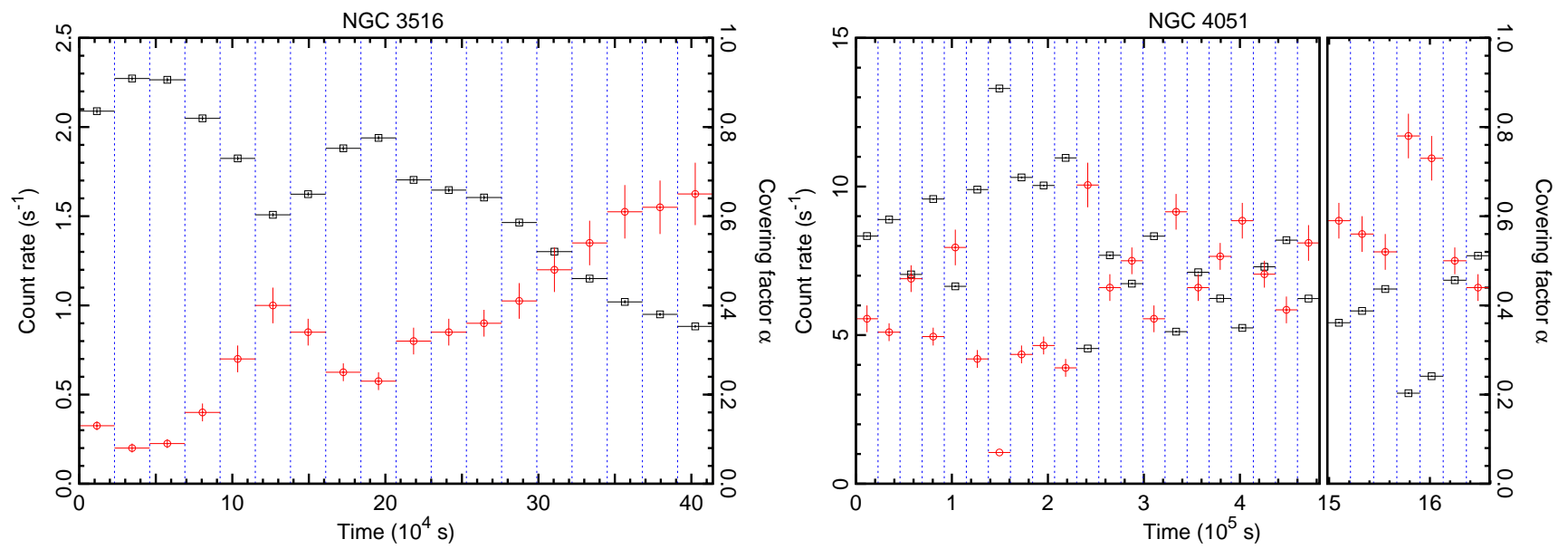

Figure 3: Variations of the observed XIS counting rates $(0.2-12 \mathrm{keV}$; black, scale in left $)$ and the partial covering fractions (red, scale in right) for NGC3516 and NGC4051.
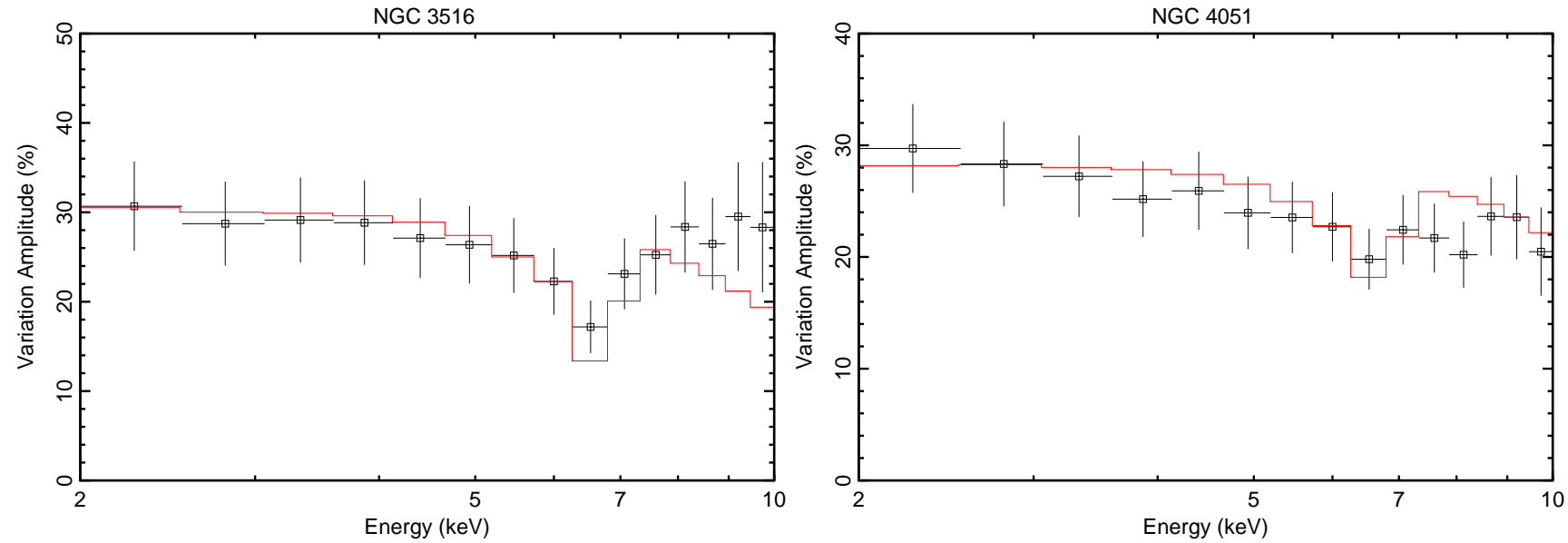

Figure 4: RMS (Root-Mean-Square) spectra for NGC3516 and NGC4051. Black points are calculated from the data, and red-histograms are calculated from the best-fit spectral models for the time-sequence spectra.

examples of the 22 RMS spectra in figure 4 . The small variability of the iron line energy band is successfully explained by the VPC model.

\section{References}

[1] Inoue, H., Miyakawa, T. \& Ebisawa, K. 2011, PASJ, 63, S669

[2] Magdziarz, P., \& Zdziarski, A. A. 1995, MNRAS, 273,837

[3] Miller, L., Turner, L. J., \& Reeves, J. N. 2010, MNRAS, 408, 1928 doi:10.1111/j.1365-2966.2010.17261.x

[4] Miyakawa, T., Ebisawa, K. \& Inoue, H. 2012, PASJ, 64, 140

[5] Zoghbi, A. et al. 2010, MNRAS, 401, 2419 doi:10.1111/j.1365-2966.2009.15816.x

\section{DISCUSSION}

MATTEO GUAINAZZI: (1) It has been recently proposed that the discovery of soft X-ray lags on time-scales consistent with reverberation from a few Schwarzschild radii constitute a very strong evidence supporting relativistic disk reflection as a driver of $\mathrm{X}$ ray variability. Can your model reproduce them? (2) Can your model put constrains on the location of the warm absorbing clouds?

KEN EBISAWA: (1) We are aware of the recent reports that several Seyfert galaxies indicate soft-lags, which some consider due to inner-disk reflection and relativistic reverberation. We have not explored yet how the observed soft-lags are explained in the scheme of our Variable Partial Covering model. [Note added in proof: 
Ken Ebisawa et al.

We noticed that the observed characteristic soft-lag is also consistent with reverberation caused by scattering X-rays passing through highly covering material, as pointed out by Miller, Turner and Reeves (2010). This seems to be a likely mechanism, since our model intrinsically requires rather large covering fractions. However, we also notice that this interpretation does not ex- plain the observed Fe $K_{\alpha}$ lags as pointed out by Zoghbi et al. (2010).] (2) Yes. From spectral variability, we estimate distance from the black hole and sizes of the warm absorbing clouds. Please find Miyakawa, Ebisawa and Inoue (2012) and Inoue, Miyakawa and Ebisawa (2011). 